\title{
Erratum
}

\section{Enantioselective Intramolecular Hydroamination of $N$-Alkenyl Ureas Catalyzed by tropos BIPHEP-Gold(I) Complexes with Au-Au Interaction}

Masafumi Kojima, Koichi Mikami* Synlett 2012, 23, 57.

In the advance (e-first) version of this article figure 1 was incomplete. The current online version has been corrected. 